\title{
Efficacy of Project-Based Instruction in a Hybridized Electronics and Con- trols Course
}

\section{Dr. John Andrew Lund, Western Washington University}

Dr. Lund's research involves the development of novel control systems, sensing and measurement tools for unique environments. His previous and ongoing research efforts include the development of a highresolution wireless instrumented mouthguard for the assessment of severity of head impacts, development of an ultra-long lifespan wireless sensor devices designed to form robust data networks in remote areas lacking infrastructure, and the development of of an electron-tunneling spectroscopy based microscope control system for molecular analysis. 


\title{
Efficacy of Project-Based Instruction in a Hybridized Electronics and Controls Course
}

\begin{abstract}
:
We present on outcome attainment from an electronics and control systems course modified to rely heavily upon a continuing project which incorporates a diverse set of educational components, including control system modeling, feedback compensation, microcontroller development, digital circuit design, digital communication, and analog circuit design. Although the majority of course outcomes saw improvement, the outcome relating to microcontroller development saw a marked reduction in outcome attainment. We present a detailed examination of this result as well as suggested remedies.
\end{abstract}

\section{Introduction:}

Engineering education has become increasingly reliant on courses that blend topics from different disciplines to prepare students for the modern engineering workforce. Sometimes this hybridization becomes formally categorized as is the case with Mechatronics. Other times courses are formed ad hoc to meet specific educational needs in a specific educational environment. Often these courses are designed to satisfy prerequisite requirements of capstone or advanced elective courses. This is a common issue identified in latter-sequence electrical engineering courses for non-EE majors, with a common solution being a collection of targeted projects with each touching on a handful of topics. ${ }^{1}$

A potential pitfall arises when courses with a specific but disparate collection of topics designed to meet the needs of subsequent courses are presented to students without proper framing. This can give the impression the content is encyclopedic in nature - part of a collection of knowledge without sequence that can be picked up or disregarded. This problem resembles concerns with project-based learning. The primary concern with project-based learning is that it runs the risk of neglecting topics that make up an essential sequence of knowledge and skill acquisition in engineering education. ${ }^{2}$

While project-based instruction runs the risk of leaving knowledge gaps across curriculum, it can serve as a tool to bridge topics and improve knowledge and skill acquisition within a course. We have adapted a course that combines control system modeling, feedback compensation, microcontroller development, digital circuit design, digital communication, and analog circuit design to include a unifying course project dependent upon these ordinarily distinct topics.

\section{Research Goals and Scope:}

The goal of this effort was to attempt to correct an identified weakness in a course offering and identify the degree to which a proposed structural change to the course corrects the weakness while maintaining student learning outcome attainment. Upon subsequent assessment, a specific 
weakness in outcome attainment was identified and we further expanded the scope of this research effort to better characterize the weakness and help formulate a proposed correction. Herein we describe the course offering prior to modification, the nature of the course project we developed, outcome attainment before and after the project addition, and a detailed assessment of one identified weakness resulting from the modification. Lastly, we propose modification which may help minimized the identified weakness.

\section{Background:}

At Western Washington University we offer a Bachelor of Science in Manufacturing Engineering degree which includes a 2-course electrical engineering support sequence (10 weeks each). The first course in the sequence covers basic circuit design and analysis, basic electronics, and basic digital design. The second course expands upon the topics covered in the first, and adds PLC and microcontroller development, as well as an introduction to control systems and motors.

\begin{tabular}{|l|l|l|}
\hline $\begin{array}{l}\text { EE 351: Electronics for } \\
\text { Engineering topics }\end{array}$ & & $\begin{array}{l}\text { EE 352: Introduction to } \\
\text { Automation and Control topics }\end{array}$ \\
\hline Circuit design and analysis & & Motors \\
\hline Operational amplifiers & & PLCs \\
\hline Electronics (diodes, transistors) & $\rightarrow$ & Voltage regulators \\
\hline Boolean Logic & $\rightarrow$ & Rotary Encoders \\
\hline Combinatorial Logic & $\rightarrow$ & Sequential Logic \\
\hline & & Microcontrollers \\
\hline & & Control Systems \\
\hline
\end{tabular}

Table 1: Basic structure of the two course EE sequence for non-EE majors

As shown in Table 1, the second EE course both builds off the previous course, as well as introducing topics quite separate from a traditional electrical or electronics topic sequence. The reason the first course is kept somewhat generic with light coverage of three distinct topics (circuits, electronics, digital systems) is because it serves a wide population of non-EE students. The second course in the sequence is almost exclusively manufacturing students who rely on the course to prepare them both for their capstone project and for a subsequent robotics elective.

Previous offerings (Spring 2015 and Spring 2014) of EE 352 followed a traditional structure of 3 hours of lecture and 2 hours of lab each week, with separate labs covering recent lecture topics. The course culminated with a 3-week final project. Upon completion of the course, students were asked to complete both a formal and informal survey. Student discontent with the structure of the course was unanimous, and summarized in the following representative comments:

The class felt like five little mini two week classes forced together.

The class is very disorganized. Topics are unrelated. Very difficult to follow from week to week. 


\section{I liked that we got to build something in this class but the project didn't seem to have anything to do with what we talked about in lecture.}

This is all very accurate and very fair criticism. The class largely prepares students for future robotics and/or capstone projects. The breadth of such courses and projects cannot be replicated as a project alongside 10 weeks of instruction. However, we felt we could improve student satisfaction with the course by developing a new course project. Previous course projects were limited to 3 weeks, which was insufficient to include implementation of rotary encoders to motor-based projects. Previous projects relied heavily on stepper motors for position control without feedback; however, the bulk of the discrepancy between project topic coverage and course topic coverage was material designed to prepare students for encoder-based motor control. Expanding the scope of the course project necessitated widening the project timeline. Noting the need to engage students with the project for the majority of the course, we decided to simply make the project an integral part of course instruction, and ensure project categories were well-mapped to course outcomes both temporally and conceptually. It should be noted that our implementation is not pure project-based learning, but also incorporates substantial "scaffolding" 3 in the form of associated instruction and guidance.

\section{Project Description:}

The two course offerings prior to the project modification described in this work relied on two separate 3-week course projects:

Syringe Pump (Spring 2014):

This was a microcontroller-based project to develop a syringe pump where a stepper motor drives a screw to depress the plunger of a syringe pump. The user can increase or decrease the volume of dispensed liquid and press a button to start the automatic dispensing process. When the syringe reaches its end, the syringe is retracted and refilled from a bottle using a system of check valves. Both the programmed volume setpoint and the status of dispensing are displayed on a seven-segment display.

Magnetic North Follower (Spring 2015):

Much like the previous project, this was a microcontroller-based project, but eliminated a user interface (no button presses or seven-segment display) and instead relied on sensor measurements and a control algorithm to drive a car towards magnetic north using a digital $\left(\mathrm{I}^{2} \mathrm{C}\right)$ compass. Although it would be possible to develop non-microcontroller-based projects, the ubiquity and low cost of microcontrollers, as well as their proven benefits ${ }^{4,5}$ for engineering instruction, led us to restrict our attention to microcontroller-based projects.

Neither of these projects were able to implement any form of motor control with feedback due to time constraints. We developed a replacement project which incorporated motor position control 
using an optical encoder. As shown in Figure 1, the newly-developed project was based on a custom printed circuit board (PCB) platform. The development platform was based on the standard Arduino header footprint, making it compatible with any microcontroller development tool based on the Arduino standard "shield" pinout, including offerings by microcontroller manufacturers such as STMicroelectronics.

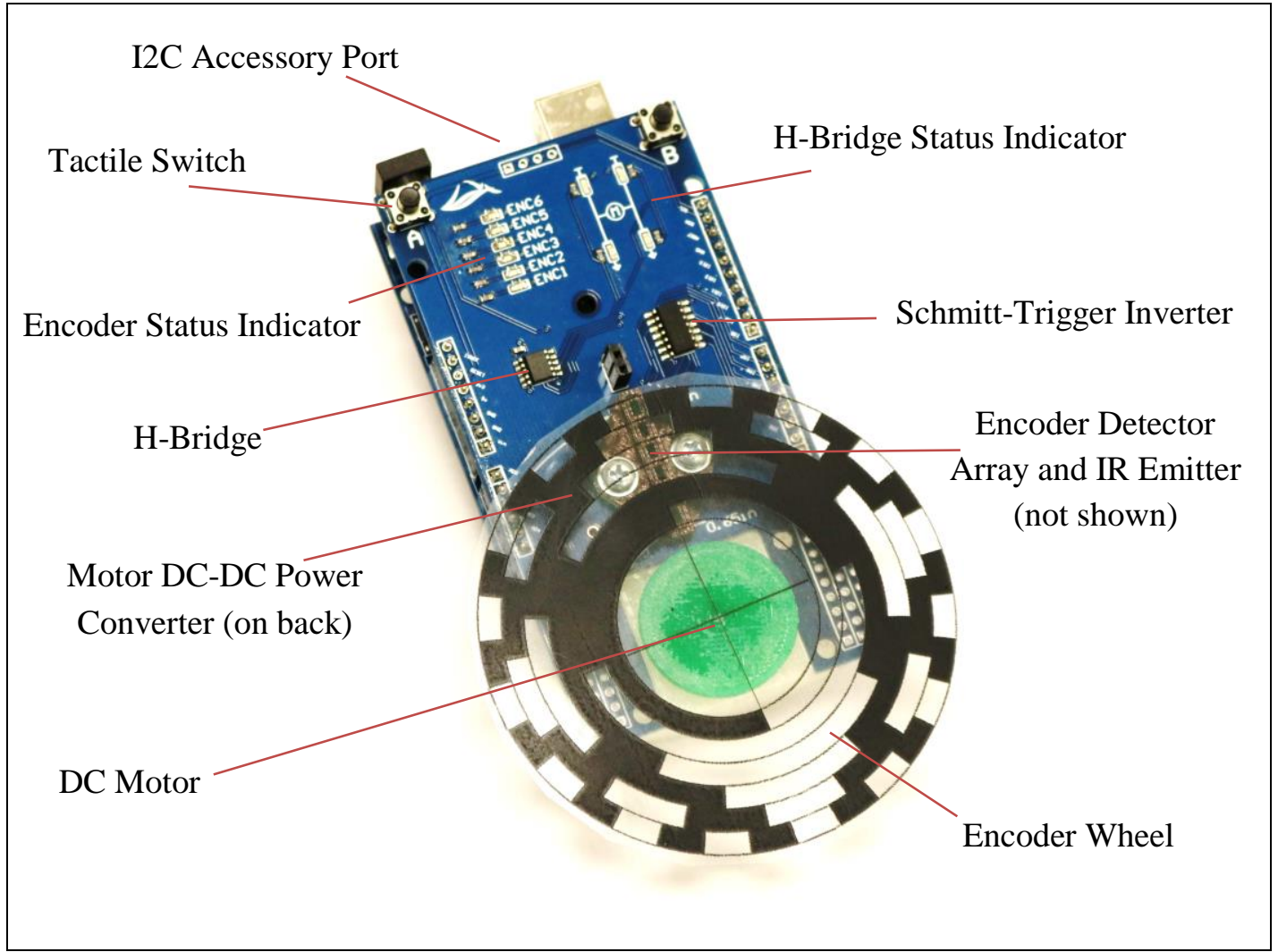

Figure 1: Image of the motor controller project platform showing components and associated course topics.

The motor controller project we developed, fundamentally, is a simple DC motor controller with optical encoder feedback and PID compensation. The project also includes additional elements to meet course outcomes, such as a DC-DC regulator which students must implement to meet the power requirements of their particular DC motor.

The problem students needed to solve was presented with two different options. Students were presented the problem in the first week of the course. The problem descriptions were:

1) "You are an unscrupulous game show host who wishes to develop a prize wheel which appears to spin with a decreasing angular velocity until naturally settling on a random prize. However, you want to rig the prize wheel so that you control whether a contestant will win an expensive prize. You must develop a system with two buttons. Pressing one 
button will cause the prize wheel to spin and never land on an expensive prize. Pressing the other button will cause the wheel to land on an expensive prize."

2) "You are a paint specialist who needs to be able to quickly identify colors and represent them by color code. You wish to design a device with a digital color sensor that measures a color, then physically rotates a color wheel to indicate the matching color. The color wheel must move from one identified color to another with a constant angular acceleration and deceleration."

The differences between the projects are largely aesthetic. The "prize wheel" project has slightly more focus on programming with random numbers, and the "color wheel" project has additional requirements for interfacing with a digital sensor. A graphic showing the sub-tasks involved in completing these projects is shown in figure 2. We elected to rely on a custom PCB as the platform for this project, but that decision presents notable tradeoffs. The downside of using a custom PCB is the design is relatively fixed, limiting student design flexibility. However, the only essential components fixed by the decision to use a PCB-based approach are the H-bridge motor driver and the Schmitt-trigger inverter. However, for the sake of convenience, the project would have limited students to one choice of H-bridge anyway, and wiring would ultimately have been identical. The main advantage of using a PCB is the resulting project is portable and inexpensive ( $<\$ 5$ total cost) so students end their project with a "totem" which is one of the fundamental elements of project-based instruction. ${ }^{8}$

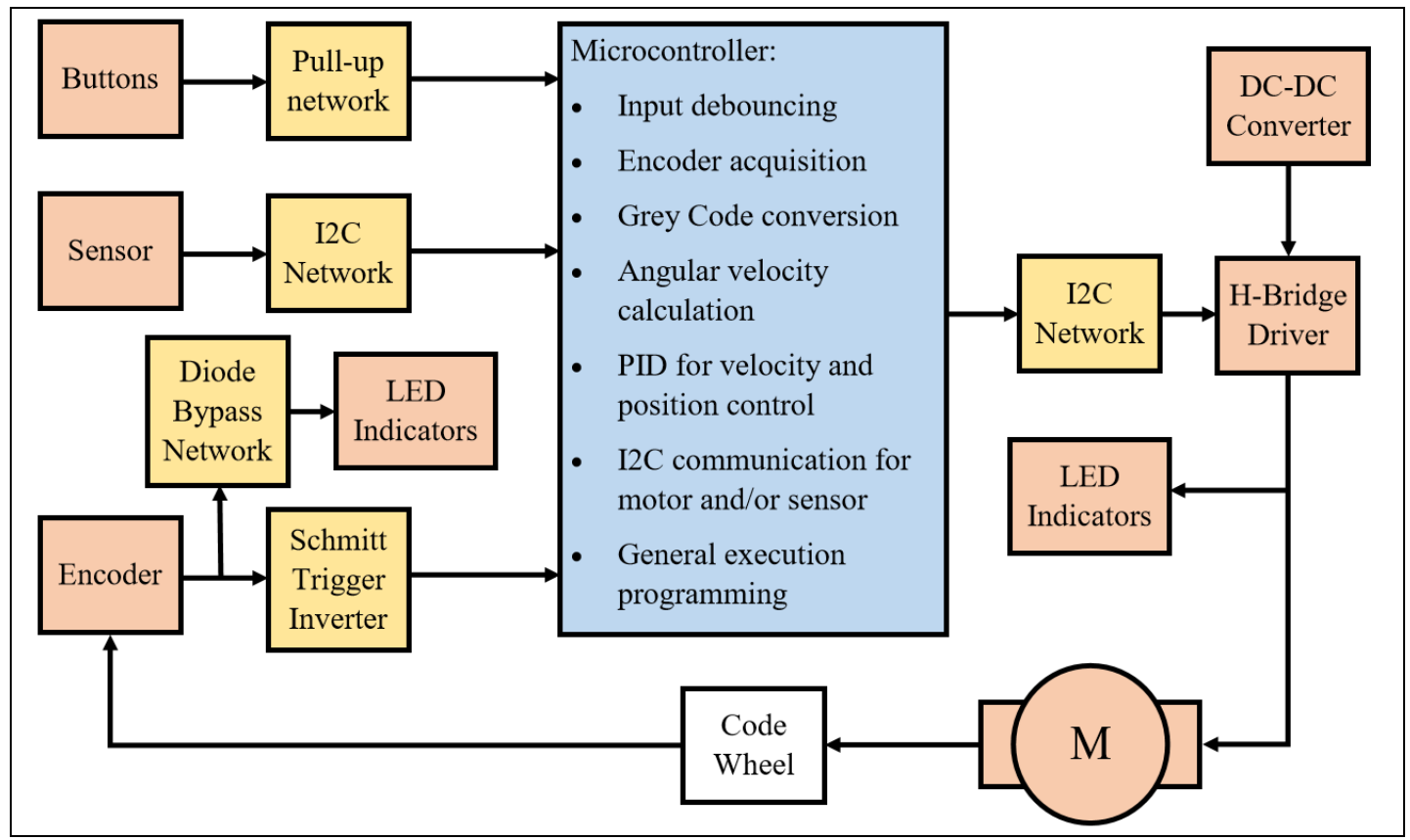

Figure 2: Project sub-tasks and associated relationships. 
As shown in Figure 2, the motor controller project includes a substantial number of sub-tasks which touch on a broad collection of course topics listed in Table 1. The specific mappings of covered topics in the motor controller project vs. the two previous course projects are shown in Table 2 .

\begin{tabular}{|c|c|c|c|c|c|c|c|c|}
\hline & $\frac{\infty}{\stackrel{0}{0}}$ & $\bigcup_{0}^{n}$ & 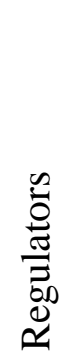 & 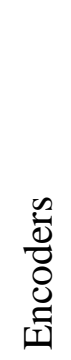 & 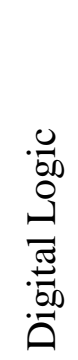 & 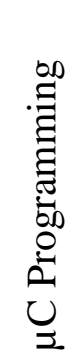 & 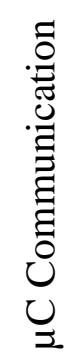 & 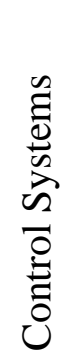 \\
\hline Automatic Syringe Pump & $\mathrm{X}$ & & & & $\mathrm{X}$ & $X$ & $\mathrm{X}$ & \\
\hline Magnetic North Follower & $\mathrm{X}$ & & & & & $\mathrm{X}$ & $\mathrm{X}$ & $\mathrm{X}$ \\
\hline Motor Controller Platform & $\mathbf{X}$ & & $\mathbf{X}$ & $\mathbf{X}$ & $\mathbf{X}$ & $\mathbf{X}$ & $\mathbf{X}$ & $\mathbf{X}$ \\
\hline
\end{tabular}

Table 2: A comparison of previous 3-week course projects with the 8-week project developed to more fully incorporate course content.

\section{Assessment Methods and Results:}

The goal of this work was not only to restructure an electrical/electronics/control system engineering class to help instill an understanding of the relationship between seemingly disconnected topics, but also to ensure outcome attainment is maintained or improved. To asses this, we compared attainment of individual outcomes between the Spring 2015 course offering ("magnetic north follower" project) and the Spring 2016 course offering ("motor controller" project). We have excluded the Spring 2014 course offering from the comparison because course outcomes were modified between 2014 and 2015.

The specific outcomes used for comparison were:

\begin{tabular}{|l|l|}
\hline$\#$ & Student Learning Outcome: \\
\hline 1 & $\begin{array}{l}\text { Design and analyze simple analog and digital automation circuits with time- } \\
\text { varying inputs. }\end{array}$ \\
\hline 2 & Use a microcontroller to engineer a basic control or automation solution. \\
\hline 3 & $\begin{array}{l}\text { Use a programmable logic controller and programming tools to develop a } \\
\text { control or automation system. }\end{array}$ \\
\hline 4 & $\begin{array}{l}\text { Determine the appropriateness of microcontrollers or PLCs based on realistic } \\
\text { design constraints. }\end{array}$ \\
\hline 5 & Design and analyze simple control systems including PID control. \\
\hline 6 & Understand the operation and application of brushed DC and stepper motors. \\
\hline 7 & Design motor feedback and control systems using rotary encoders. \\
\hline 8 & $\begin{array}{l}\text { Be able to test and troubleshoot circuits using the appropriate } \\
\text { instrumentation. }\end{array}$ \\
\hline
\end{tabular}


Evaluations were based upon scoring of specific exam questions which were consistent between the two course offerings and graded based on a consistent rubric. The 2015 offering included a total of 15 students whose average outcome assessment scoring was used for the analysis. The 2016 offering included 19 students; however, 2 elected to do a custom project (digital compass wheel) which was related but not reported here, so scoring for these students is excluded from the analysis.

The results of the assessment analysis are compared in Figure 3. As shown, there was a marginal improvement or no notable change across the board with the exception of a $12 \%$ reduction in score on Outcome 2 and a 10\% improvement in score for Outcome 5. The reduction in student learning outcome attainment for the outcome related to the use of a microcontroller was surprising, given the heavy reliance upon microcontroller development in the project.

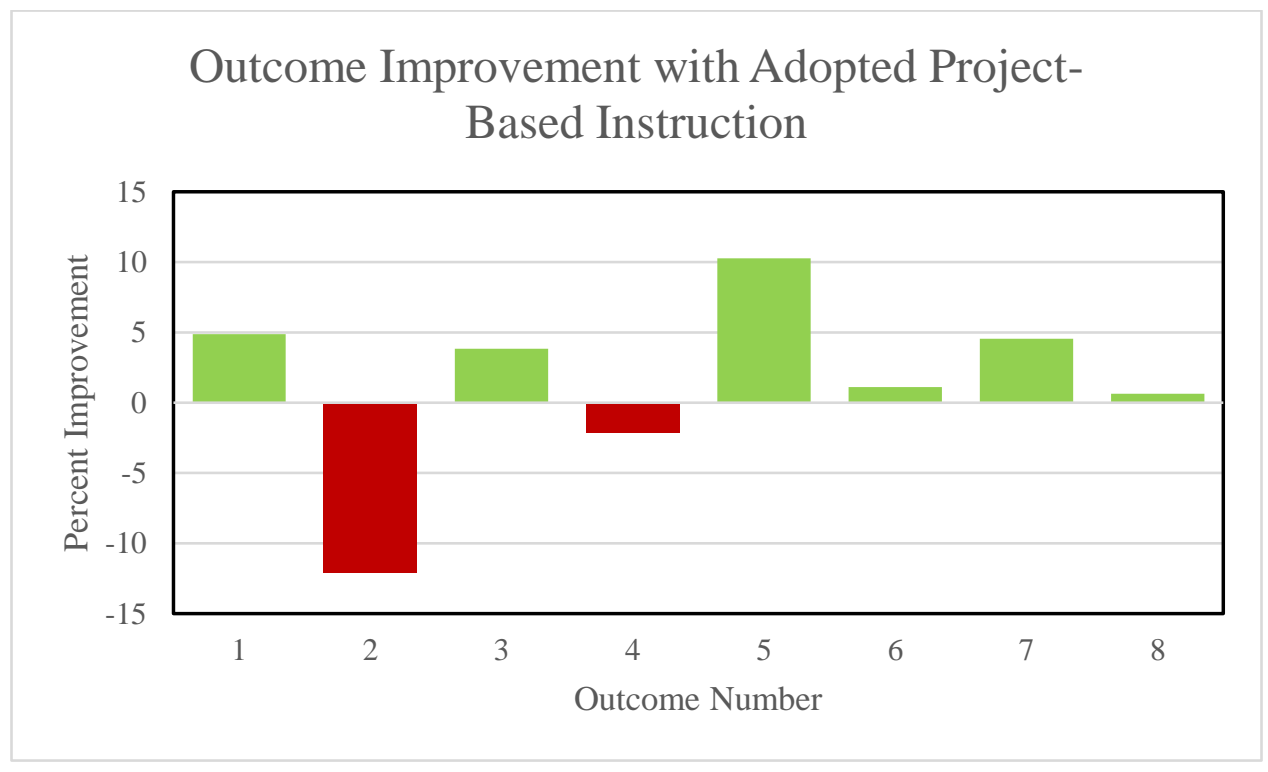

Figure 3: Percent change in average outcome score with incorporation of an 8week project component

Deficiency in microcontroller development skills was also noted during final project oral examinations. The subjective assessment was that some students working with partners (each student had to complete their own project, but partners were allowed for programming portions due to limitations of one computer per lab bench) relied heavily on their partner's contribution to microcontroller programming tasks. Based on this observation, a survey was distributed to individuals who worked with partners on programming portions of the project. Students were told their responses would be kept private, and would not have any impact on anyone's grade (including their own). Students were asked to rate the contribution of their partner(s) on the project programming and the rated contributions (including self-ratings) were compared to their individual Outcome 2 scoring. Figure 4 shows the results of this survey, suggesting a relationship between students with lacking contributions to group programming and poor 
outcome attainment related to use of microcontrollers. It should also be noted that in the 2015 outcome assessment, no individual students scored below a 10 out of 20 in Outcome 2 attainment, whereas 4 students scored below a 10 out of 20 in 2016, all of whom worked with partners for project programming. This is a commonly-observed effect and one of the primary disadvantages of team-based learning. ${ }^{6}$ Qualitative course assessment based on student feedback also indicates a general dissatisfaction with the programming difficulty of the project. Student feedback also indicated a general preference for PLC development versus microcontroller development. The topic of PLCs was the only category of instruction not connected to the project. PLC instruction consisted of 2 week of instruction and laboratory exercises. Many students expressed more confidence in their ability to develop ladder logic programs than their ability to program systems using $\mathrm{C}$.

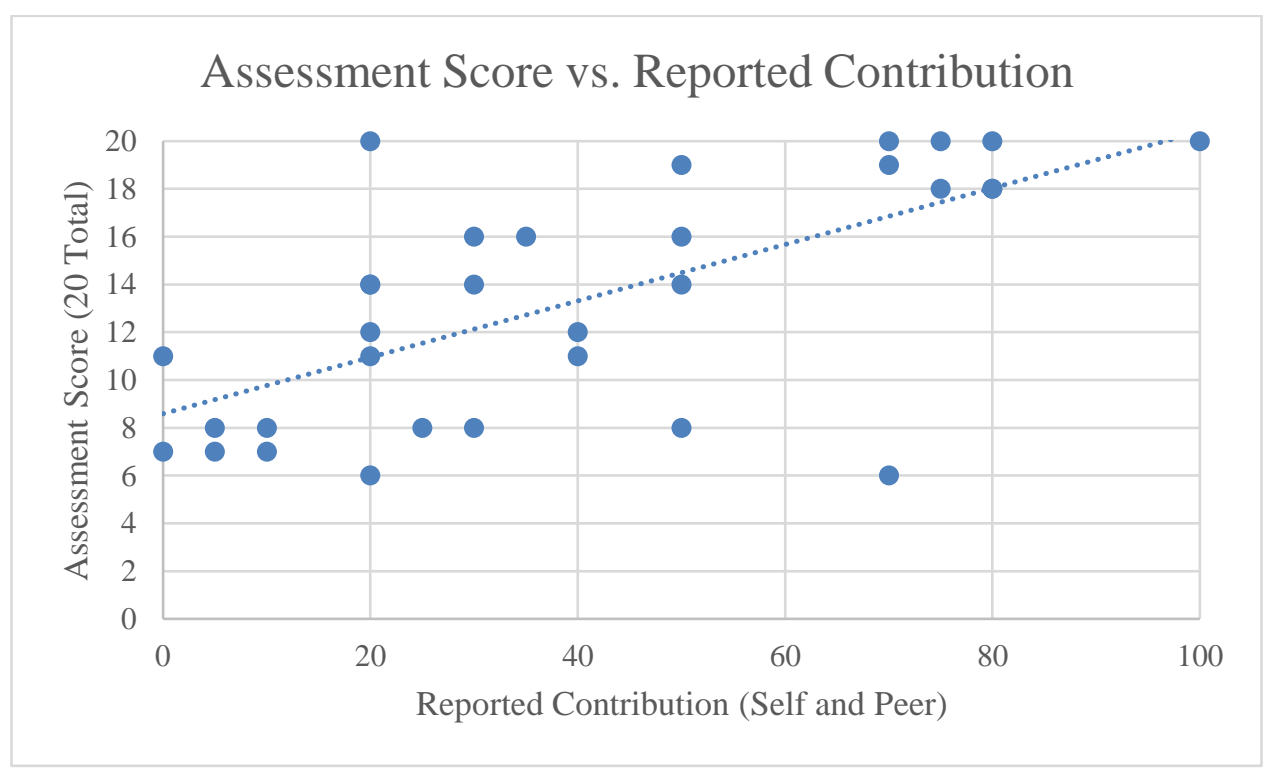

Figure 4: Comparison of individual assessment score with peer and self-reported contribution for students working in groups on project programming.

\section{Discussion:}

Course outcome evaluation indicates the adaptation of a project component for the course resulted in a marginal improvement or no change in student learning outcome attainment with the exception of microcontroller development competency, which saw a notable outcome attainment reduction. Further analysis suggests the cause of this reduction may be related to an un-equal laboratory experience for students partnering to complete microcontroller programming tasks. Based on these results, we propose three possible corrections to improve outcome assessment:

1) Implement low-level functions for students. Timer and interrupt-based functions necessary for implementing encoder-based position and velocity control seemed to be 
exceptionally difficult for students, many of whom seemed to give up and lean on their partner to identify a solution.

2) Require students to develop their microcontroller programs independently. With reasonable provision of low-level code, the programming task should be manageable even for lower-performing students. An alternative approach is to use a tool such as intermediate process assignments to address and correct unequal distribution of participation in groups and correct the imbalance. ${ }^{7}$

3) Reduce PLC focus. The course flow introduces PLCs early (before microcontrollers) and spends substantial class an laboratory time on this topic, perhaps over-emphasizing one particular method of program development to which many students seem drawn to use, requiring a conceptual restructuring to migrate to a sequential programming language like C. Moving coverage of PLCs to after implementation with microcontrollers could help improve student outcome attainment.

\section{Conclusion:}

We have presented an evaluation of the efficacy of an electrical engineering course tailored for non-EE majors from primarily lab and lecture based learning to a strongly project dependent format. We have described the means by which this change was implemented, and reported on the resulting change in outcome attainment. As part of this evaluation process, we identified a deficiency resulting from the course adaptation and, based on further objective and subjective assessment, suggest modification to mitigate these unintended outcome impacts. These suggested improvements could benefit future implementations of the course and serve as guidance for anyone wishing to emulate this approach for engineering courses that are composed of disparate instructional topics. 


\section{References:}

1. “Teaching Engineering To Non Electrical Engineering Majors," ASEE Annual Conference 1998, https://peer.asee.org/7451

2. Thomas, J. W. A Review of Research on Project-Based Learning. (2000)

3. Guzdial, M., "Technological support for project based learning." In C. Dede (Ed.), 1998.

4. Learning with Technology, (pp. 47-72), Alexandria, VA: ASCD. Giurgiutiu, V., Lyons, J., Rocheleau, D., and Liu, W., Mechatronics/microcontroller Education for Mechanical Engineering Students at the University of South Carolina, Mechatronics, 2005, 15(9): pp. 1025-1036.

5. Saad, A., "A Project-based Approach to Learning Digital Logic Design Using Simple Mobile Robots," in Proc. Int'l. Conf. on Eng. Educ., Valencia, Spain, 2003.

6. Michaelsen, L. K., Bauman Knight, A., Fink, L. D., Team-Based Learning: A Transformative Use of Small Groups in College Teaching, Sterling, VA: Sylus, 2004.

7. Barkley, E.F., Cross, K.P., and Major, C.H. "Collaborative learning techniques: A handbook for college faculty." San Francisco: Jossey-Bass, 2005.

8. Krajcik, J. S., \& Blumenfeld, P. C. (2006). Project-based learning (pp. 317-34). na. 